\section{Change in Velocity of Light emitted by a Moving Source}

Some interest has been aroused recently by a paper by W. Kantor ${ }^{1}$, describing an experiment to detect the change in the velocity of light emitted by a moving source. The author explains his results on the hypothesis that the velocity of light as it leaves the moving surface of a glass block through which it has been transmitted is given by the expression $c^{\prime}=c+\rho v$, where $c$ is the usual velocity of light, $v$ the velocity of the glass surface in the direction of motion of the light beam, and $\rho$ is some constant, equalling 1 on classical theory and 0 according to relativity. The experiment described then shows an effect which can be explained by assuming $p=2 / 3$.

We have recently constructed an apparatus to test this hypothesis. White light is passed through a slit and is then collimated. It then passes through a semi-circular disk of glass which is rotated by a high-speed electric motor about an axis in its own plane, the axis of rotation being normal to the direction of motion of the light beam. It is then possible to show quite simply on the foregoing hypothesis that the wave front emerging from the apparatus will be moving more slowly on one side than on the other, and so should rotate as it is propagated.

The light was received in a telescope which could be placed at different distances from the apparatus, and the deviation of the light could then be detected by comparison with the image which had not passed through the disk. The angle of deviation is $\theta=\rho w x / c$, where $w$ is the angular velocity of the glass disk, and $x$ is the distance of the telescope from the disk.

Under the conditions of our experiment $w=1,500$ $(=14,300$ r.p.m. $), x$, the distance of the telescope from the disk $=20 \mathrm{~m}$, and $c=3 \times 10^{10} \mathrm{~cm} / \mathrm{sec}$, so that if $p=1$, $\theta=20 \mathrm{sec}$ of arc. This is of course easily detectable in a moderate-sized telescope, and in our case the minimum angle detectable was $0.5 \mathrm{sec}$, the limit being set by the size of the diffraction pattern seen in the telescope.

At no time was any deviation of the light observed and we conclude that $\rho$ in this case is less than 0.025 , if indeed it is different from zero.

Special relativity predicts a small effect of $0.0008 \mathrm{sec}$ of arc due to Fresnel drag in the glass, but this deviation is too small to be detected by this technique.

\section{J. F. JAMES}

Physical Laboratories,

University of Manchester.

${ }^{1}$ Kantor, W., J. Opt. Soc. Amer., 52, 982 (1962).

\section{Sub-sieve Sizing by Sedimentation Balance}

IN determining the size distribution of particles in samples of finely ground barytes, difficulties have been experienced in determining experimentally the ultimate balance reading corresponding to sedimentation equilibrium $^{1}$. It takes an inordinately long time for equilibrium to be attained, and even then this does not necessarily represent complete sedimentation of all solid particles. Consequently, even at equilibrium the balance reading $\left(S_{\infty}\right)$ will be less than the theoretical maximum reading corresponding to the weight of solid introduced $\left(S_{m}\right)$.

In these circumstances, sub-sieve sizing by sedimentation balance can become a protracted and inconvenient operation if the conventional gravimetric methods of determining $S_{\infty}$ and $S_{m}$ are adopted. However, some preliminary success has been achieved in these laboratories by using forms of exponential functions to determine $S_{\infty}$, permitting termination of the experiment long before sedimentation equilibrium is attained and also obviating any need to evaporate liquid and weigh dry samples.

It is our experience that in many cases the upper 10-20 per cent of the sedimentation curve can be described by the equation:

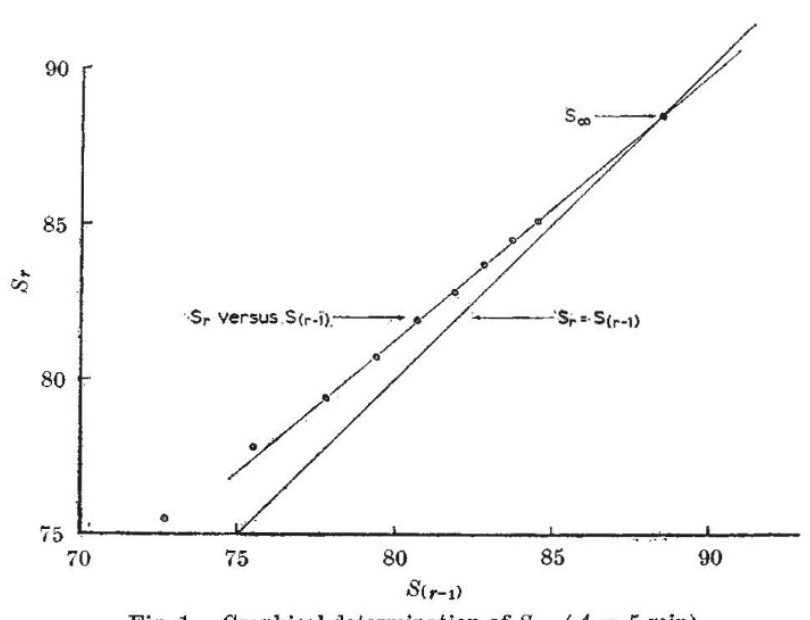

Fig. 1. Graphical determination of $S_{\infty}(\Delta=5 \mathrm{~min})$

$$
S=S_{\infty}\left(1-\theta^{-b t}\right)
$$

where $t$ is time, $S$ is the balance reading and $b$ is a rate constant.

This equation may be represented by the pair of equations:

and

$$
t_{r}=t_{(r-1)}+\Delta
$$

$$
S_{r}=S_{(r-1)} \cdot e^{-b \Delta}+S_{\infty}\left(1-e^{-b \Delta}\right)
$$

where $r$ is an integer and $\Delta>0$ and is constant ${ }^{2}$.

A solution of equation (3) is $S_{(r-1)}=S_{r}=S_{\infty}$, and by plotting $S_{r}$ against $S_{(r-1)}, S_{\infty}$ is readily obtained; $b$ is not of significance in this instance. The experimental results given in Table 1 are plotted in this manner in Fig. 1; the point of intersection of the experimental line, $S_{r}$ versus $S_{(r-1)}$, with the theoretical line, $S_{r}=S_{(r-1)}$, is the value of $S_{\infty}$.

In Table 1, the times from 5 min onwards advance in arithmetic progression in accordance with equation (2). The usual experimental technique is to take balance readings at times advancing in geometric progression; in this case, suitable values for plotting according to equation (3) are obtained by graphical interpolation. Additionally, interpolation has the advantage of smoothing instrument

\begin{tabular}{|c|c|c|c|}
\hline Time $(\min )$ & Scale reading $(S)$ & Time (min) & Scale reading $(S)$ \\
\hline $\begin{array}{r}0 \\
1 \\
2 \cdot 5 \\
5 \cdot 0 \\
7 \cdot 5 \\
10 \cdot 0 \\
15 \cdot 0 \\
20.0 \\
25 \cdot 0\end{array}$ & $\begin{array}{r}0 \cdot 8 \\
4 \cdot 8 \\
14 \cdot 8 \\
31 \cdot 2 \\
43 \cdot 7 \\
52 \cdot 2 \\
62 \cdot 3 \\
68 \cdot 3 \\
72 \cdot 7\end{array}$ & $\begin{array}{l}30 \cdot 0 \\
35 \cdot 0 \\
40 \cdot 0 \\
45 \cdot 0 \\
50 \cdot 0 \\
55 \cdot 0 \\
60 \cdot 0 \\
65 \cdot 0 \\
70 \cdot 0\end{array}$ & $\begin{array}{l}75 \cdot 5 \\
77 \cdot 8 \\
79 \cdot 4 \\
80 \cdot 7 \\
81 \cdot 9 \\
82 \cdot 8 \\
83 \cdot 7 \\
84 \cdot 5 \\
85 \cdot 1\end{array}$ \\
\hline
\end{tabular}
reading errors. Having determined $S_{\infty}$, the size distribution is determined in the usual manner ${ }^{3}$.

This procedure has been used successfully in this Department to analyse a number of sedimentation balance curves and has also been used in other similar applications ${ }^{4}$. The curves were related to separations according to particle size in a sedimentation classifier, and it has been logical throughout to relate sizing analyses to that proportion of the solid in suspension which would settle; that is, to $S_{\infty}$ and not $S_{m}$. Results obtained by other workers have been examined successfully by the same procedure.

In some instances the plot of $S_{r}$ versus $S_{(r-1)}$ does not give a straight line, but the curve obtained would probably fit another function having an unknown asymptote. In the case illustrated, the plot is linear over the last 12 per cent of the sedimentation curve; but curvature becomes 